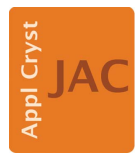

JOURNAL OF

APPLIED

CRYSTALLOGRAPHY

ISSN 1600-5767

Received 16 December 2016

Accepted 18 August 2017

Edited by E. P. Gilbert, ANSTO, Kirrawee DC, Australia

Keywords: spin-echo small-angle neutron scattering; small-angle neutron scattering; data analysis.

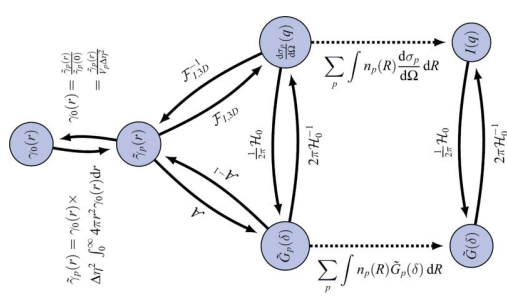

C 2017 International Union of Crystallography

\section{Transformation cycle between the spherically symmetric correlation function, projected correlation function and differential cross section as implemented in SASfit}

\author{
J. Kohlbrecher ${ }^{\mathrm{a} *}$ and A. Studer ${ }^{\mathrm{b}}$
}

aLaboratory for Neutron Scattering and Imaging (LNS), Paul Scherrer Institute, CH-5232 Villigen PSI, Switzerland, and
bScientific Computing, Paul Scherrer Institute, CH-5232 Villigen PSI, Switzerland. *Correspondence e-mail:
joachim.kohlbrecher@psi.ch

Spin-echo-based small-angle neutron scattering techniques like spin-echo SANS (SESANS) or spin-echo modulated SANS (SEMSANS) as well as dark-field (DF) imaging are directly sensitive to $\tilde{G}(\delta)$, which is the projection of the scattering length density autocorrelation function $\tilde{\gamma}(r)$. Here, a simplified transformation cycle relating the spherically symmetric correlation function $\tilde{\gamma}(r)$, the projected correlation function $\tilde{G}(\delta)$ and the macroscopic small-angle scattering cross section $\mathrm{d} \Sigma / \mathrm{d} \Omega$ is introduced. The suggested changes to the cycle make it easier to include size distribution on an absolute scale if the data from the different techniques are fitted simultaneously. As up to now only very few analytical expressions for the projected correlation function are known, a numerical transformation of known scattering functions into the projected correlation function in the SASfit software package is supplied. Furthermore, a new analytical expression for the projected correlation function for polymers that can be described by the generalized Gaussian coil model is provided. For this polymer model, the Hankel transform used to calculate $\tilde{G}(0)$ from the SANS signal is divergent for a certain parameter range describing a polymer in a good solvent and finite in the case of a poor solvent. It is therefore a suitable example of how the experimentally available $q$ range can strongly influence the obtained results.

\section{Introduction}

In recent years, several imaging techniques using gratings and spin-echo-based small-angle neutron scattering (SANS) have been developed, which probe directly the projected correlation function $\tilde{G}(\delta)$ in real space. The spin-echo-based techniques are spin-echo SANS (SESANS) (Rekveldt, 1996; Krouglov et al., 2003; Andersson et al., 2008) and spin-echo modulated SANS (SEMSANS) (Li et al., 2016; Sales et al., 2015; Strobl, Tremsin et al., 2012; Strobl, Wieder et al., 2012; Strobl et al., 2015; Kusmin et al., 2017). Dark-field imaging techniques with grating interferometers (DF imaging) have been developed for both X-rays and neutrons (Pfeiffer, Weitkamp et al., 2006; Pfeiffer, Grünzweig et al., 2006; Grünzweig et al., 2008; Pfeiffer et al., 2008; Strobl et al., 2008, 2016; Strobl, 2014). These methods probe length scales ranging from a few tens of nanometres up to a few tens of micrometres and are therefore of interest for studying those length scales, which are at the upper limit and beyond for SANS and small-angle X-ray scattering.

In DF imaging, the normalized visibility $V_{\mathrm{s}}\left(\xi_{\mathrm{GI}}\right) / V_{0}\left(\xi_{\mathrm{GI}}\right)$ as a function of the autocorrelation length of a grating setup $\xi_{\mathrm{GI}}$ is given by (Strobl, 2014) 


$$
\frac{V_{\mathrm{s}}\left(\xi_{\mathrm{GI}}\right)}{V_{0}\left(\xi_{\mathrm{GI}}\right)}=\exp \left\{\Sigma t\left[G\left(\xi_{\mathrm{GI}}\right)-1\right]\right\}
$$

where $\Sigma t$ is the total scattering probability and $t$ the sample thickness. For SEMSANS the normalized $V_{\mathrm{s}} / V_{0}$ Michelson visibility of the fringes (Strobl et al., 2015; Li et al., 2016) depends in the same way on the projected correlation function as the visibility in DF imaging [equation (1)]. For SESANS the normalized depolarization $P_{\mathrm{s}}(\delta) / P_{0}(\delta)$ as a function of the spin-echo length $\delta$ (Rekveldt et al., 2003; Krouglov et al., 2003) also has the same dependency on the projected correlation function and is given as

$$
\frac{P_{\mathrm{s}}(\delta)}{P_{0}(\delta)}=\exp \{\Sigma t[G(\delta)-1]\},
$$

where the function $G(\delta)$ is a projected and normalized scattering length density autocorrelation function. Andersson and co-workers have given a transformation triangle between the scattering length density autocorrelation function $\gamma_{0}(r)$, its projection $G(\delta)$ and the differential scattering cross section $I(q)$. In their paper (Andersson et al., 2008) this triangle was assigned to the projection-slice theorem applied in imaging (Bracewell, 1956, 1990, 2000). In a special case, namely for circularly symmetric images, this theorem simplifies to the $\mathcal{F H} \mathcal{A}$ cycle, where $\mathcal{F}, \mathcal{H}$ and $\mathcal{A}$ are the Fourier, Hankel and Abel transforms, respectively. The $\mathcal{F H} \mathcal{A}$ cycle states that the successive application of the three transforms or a cyclic order of them yields the original function (Bracewell, 2000), i.e.

$$
\mathcal{H} \circ \mathcal{F} \circ \mathcal{A}=\mathcal{F} \circ \mathcal{A} \circ \mathcal{H}=\mathcal{A} \circ \mathcal{H} \circ \mathcal{F}=\mathcal{I} \text {. }
$$

$\mathcal{I}$ is the identity operator and $\circ$ denotes composition of operators. However, in contrast to the $\mathcal{F H} \mathcal{A}$ cycle, in spinecho-based SANS or DF imaging we do not have to apply a one-dimensional Fourier transformation in an arbitrary direction of a circularly symmetric image but rather a threedimensional Fourier transformation of a spherically symmetric function. So it seems not immediately obvious how the transformation cycle reported by Krouglov et al. (2003) and the $\mathcal{F H} \mathcal{A}$ cycle are related. By showing the mathematical relation between the two transformation cycles we can define three functions, $\tilde{\gamma}(r), \tilde{G}(\delta)$ and $I(q)$, which are only related via simple transformations without any additional scaling or normalization. The normalization and rescaling operations by the correlation length $\xi$ and volume fraction $\phi$ used by Andersson et al. (2008) become obsolete. Nevertheless, a proper consideration of concentration effects has to be included in the description of the correlation function of the sample, as done for example by Kruglov (2005) rather than by a simple scaling. The transformation cycle presented here has been tested numerically for several particle shapes including polydispersity and has been implemented in the software package SASfit (Breßler et al., 2015). By appropriate definition of $\tilde{G}(\delta)$ we can keep exactly the same interpretation for the parameters in the size distribution of polydisperse systems, which helps when comparing SANS and SESANS or SEMSANS on an absolute scale. Therefore, we also explain how this absolute scale is handled in the SASfit package.
Finally, we supply a new analytical expression of the projected correlation function $\tilde{G}(\delta)$ for the generalized Gaussian coils model of Hammouda (2012), which has a varying Porod law depending on the solvent quality. This example is used to show under which conditions the limited $q$ range covered by a detector in a SESANS experiment might become important.

\section{Autocorrelation function and differential scattering cross section on absolute scale}

The differential scattering cross section $\mathrm{d} \sigma / \mathrm{d} \Omega(q)$ normalized on the illuminated sample volume $V_{\mathrm{s}}$, which is the experimentally measured scattering intensity $I(q)$, can be calculated by the squared Fourier transform of the scattering length density contrast distribution $\Delta \eta(\mathbf{r})$ :

$$
I(\mathbf{q})=\frac{\mathrm{d} \Sigma}{\mathrm{d} \Omega}(\mathbf{q})=\frac{1}{V_{\mathrm{s}}} \frac{\mathrm{d} \sigma}{\mathrm{d} \Omega}(\mathbf{q})=\frac{1}{V_{\mathrm{s}}}\left|\int_{V_{\mathrm{s}}} \Delta \eta(\mathbf{r}) \exp (-i \mathbf{q} \cdot \mathbf{r}) \mathrm{d} \mathbf{r}\right|^{2} .
$$

By normalization of the differential scattering cross section $\mathrm{d} \sigma / \mathrm{d} \Omega$ on the illuminated sample volume $V_{\mathrm{s}}=t S$, where $S$ is the beam cross section and $t$ the sample thickness, the units of the scattering intensity $I(\mathbf{q})$ or the macroscopic cross section $\mathrm{d} \Sigma / \mathrm{d} \Omega(\mathbf{q})$ are given in reciprocal length units (Price \& Fernandez-Alonso, 2013). The differential cross section $\mathrm{d} \sigma / \mathrm{d} \Omega(\mathbf{q})$ has the same definition as $\left(\mathrm{d} \Sigma_{\mathrm{S}} / \mathrm{d} \Omega\right)(\mathbf{q})$ in the work of Rekveldt et al. (2003) and Krouglov et al. (2003). For the projected correlation function the definitions on an absolute scale are not always consistent in the literature and differ by some constant factors. We use here the definitions

$$
\begin{aligned}
\Sigma t G(\delta) & =t \lambda^{2} \tilde{G}(\delta) \\
& =\frac{t \lambda^{2}}{(2 \pi)^{2}} \int_{-\infty}^{\infty} \int_{-\infty}^{\infty} I(\mathbf{q}) \cos \left(q_{z} \delta\right) \mathrm{d} q_{z} \mathrm{~d} q_{y} \\
& =\frac{t \lambda^{2}}{2 \pi} \int_{0}^{\infty} I(q) J_{0}(q \delta) q \mathrm{~d} q=\frac{t \lambda^{2}}{2 \pi} \mathcal{H}_{0}[I(q)](\delta),
\end{aligned}
$$

$\lambda$ being the wavelength of the beam, $J_{0}$ the zero-order Bessel function and $\mathcal{H}_{0}$ the Hankel transform of zero order [see also equation (22)]. Furthermore in equation (7) an isotropic scatterer is assumed. $G(\delta)$ is the normalized projected correlation function so that $G(0)=1$. $\tilde{G}(\delta)$ as used here and by Rekveldt et al. (2003) differ by the factor $t \lambda^{2}$ to make it independent of instrument parameters and only dependent on sample properties. The total scattering probability $\Sigma t$ covered by the detector,

$$
\Sigma t=\int_{\text {detector }} \frac{1}{S} \frac{\mathrm{d} \sigma(q)}{\mathrm{d} \Omega} \mathrm{d} \Omega=\int_{\text {detector }} t \frac{\mathrm{d} \Sigma(q)}{\mathrm{d} \Omega} \mathrm{d} \Omega,
$$

has a well defined meaning if the intensity covered by the detector is practically the same as that scattered by the sample in $4 \pi$, i.e. the main scattering from the sample has to be in the forward direction. However, for slowly decaying form factors 
like those of polymers, which are discussed later on in the manuscript, this is no longer necessarily the case. For those samples also a certain fraction of the scattering can happen outside the solid angle covered by the detector. In these cases it is an advantage not to introduce parameters that can be calculated only by integration of a model over the whole $q$ space and might even be undefined in certain circumstances. Furthermore, by avoiding normalization we can perform the integration directly over a size distribution with the correct weighting of differently sized particles, both for the differential scattering cross section and for the projected correlation function. Therefore the transformation triangle presented here has been formulated without normalization by parameters like the correlation length $\xi$, volume fraction $\phi$ or total scattering probability $\Sigma t$ as normally done.

For isotropic media, which we will consider in the following, we have

$$
\begin{aligned}
I(q) & =\frac{1}{V_{\mathrm{s}}}\left|\int_{0}^{\infty} \Delta \eta(r) \frac{\sin q r}{q r} 4 \pi r^{2} \mathrm{~d} r\right|^{2} \\
& =\frac{1}{V_{\mathrm{s}}} \int_{0}^{\infty} \tilde{\gamma}(r) \frac{\sin q r}{q r} 4 \pi r^{2} \mathrm{~d} r=\frac{1}{V_{\mathrm{s}}} \mathcal{F}_{\mathrm{I}, 3 \mathrm{D}}[\tilde{\gamma}(r)](q) .
\end{aligned}
$$

$\mathcal{F}_{\mathrm{I}, 3 \mathrm{D}}$ denotes the three-dimensional Fourier transform operator of a spherically symmetric function (index I denotes isotropic) and $\tilde{\gamma}(r)$ the spherically symmetric scattering length density contrast autocorrelation function, which is given by

$$
\tilde{\gamma}(r)=\int_{V_{\mathrm{s}}} \Delta \eta\left(\mathbf{r}^{\prime}\right) \Delta \eta\left(\mathbf{r}^{\prime}+\mathbf{r}\right) \mathrm{d} \mathbf{r}^{\prime} .
$$

For spherically symmetric objects this correlation function only depends on the modulus $r=|\mathbf{r}|$.

SASfit is designed to describe the scattering of the sample via the known scattering behaviour of single particles. In the simplest case of non-interacting particles both the scattering curve and the correlation function of the whole sample are simply the linear combination of the differential scattering cross section $\left(\mathrm{d} \sigma_{p} / \mathrm{d} \Omega\right)(q)$ and correlation function $\tilde{\gamma}_{p}(r)$ of the individual particles $p$, i.e. for $N$ identical spherically symmetric particles $(\mathrm{d} \sigma / \mathrm{d} \Omega)(q)=N\left(\mathrm{~d} \sigma_{p} / \mathrm{d} \Omega\right)(q)$ and $\tilde{\gamma}(r)=N \tilde{\gamma}_{p}(r)$.

For higher volume fractions the correlation function of the sample is no longer simply the sum of the correlation functions of the individual scatterers in the beam. Kruglov has shown how the correlation function has to be extended for colloids with known interaction potential (Kruglov, 2005; Li et al., $2010,2011)$. If the interparticle correlations are included, the additional factor $(1-\phi)$ reflecting the Babinet principle in scattering comes out automatically without introducing any scaling. The correlation function per particle for a concentrated system of identical interacting colloidal particles is given by (Kruglov, 2005; Li et al., 2011)

$$
\frac{1}{N} \tilde{\gamma}(\mathbf{r})=\left(\delta_{\mathrm{D}} * \tilde{\gamma}_{p}\right)(\mathbf{r})+n\left[(g-1) * \tilde{\gamma}_{p}\right](\mathbf{r}),
$$

where $*$ denotes the convolution operator of two functions $f_{1}$ and $f_{2}$, i.e. $\left(f_{1} * f_{2}\right)(\mathbf{x}):=\int_{\mathbb{R}^{3}} f_{1}(\tau) f_{2}(\mathbf{x}-\boldsymbol{\tau}) \mathrm{d} \boldsymbol{\tau}$, and $\delta_{\mathrm{D}}(\mathbf{r})$ is the
Dirac delta function. For spherically symmetric potentials, $g(r)$ is the radial distribution function, which can be calculated by solving the Ornstein-Zernike equations (Caccamo, 1996; Bomont, 2008). $n=N / V_{\mathrm{s}}$ is the particle number density. A numerical algorithm to solve the Ornstein-Zernike equations for monodisperse particles for several potentials and closure relations is also available in the $S A S f i t$ software package. Even in the case of a simple size distribution the problem becomes immediately nonlinear and partial structure factors have to be calculated (Vrij, 1979), which does not match into the present numerical framework of the SASfit package. Nevertheless, the transformation triangle is always valid. If for high volume fractions interparticle correlations are taken into account assuming monodisperse particles the implemented averaging over the size distribution would correspond to the 'local monodisperse approximation' (Pedersen, 1994).

To see this one takes the Fourier transform $\mathcal{F}_{\text {I,3D }}$ of equation (12) and obtains

$$
\begin{aligned}
\mathcal{F}_{\mathrm{I}, 3 \mathrm{D}}[\tilde{\gamma}(r) / N](q)= & \mathcal{F}_{\mathrm{I}, 3 \mathrm{D}}\left[\tilde{\gamma}_{p}(r)\right](q) \\
& +\mathcal{F}_{\mathrm{I}, 3 \mathrm{D}}\left[\tilde{\gamma}_{p}(r)\right](q) \mathcal{F}_{\mathrm{I}, 3 \mathrm{D}}[n\{g(r, \phi)-1\}](q) \\
= & \frac{\mathrm{d} \sigma_{p}}{\mathrm{~d} \Omega}(q)+\frac{\mathrm{d} \sigma_{p}}{\mathrm{~d} \Omega}(q)[S(q, \phi)-1] \\
= & \frac{\mathrm{d} \sigma_{p}}{\mathrm{~d} \Omega}(q) S(q, \phi)
\end{aligned}
$$

for concentrated monodisperse colloidal systems having a structure factor $S(q, \phi)$. A subsequent averaging over a size distribution then corresponds to the local monodisperse approximation. By including interparticle interactions the scattering invariant of $n\left(\mathrm{~d} \sigma_{p} / \mathrm{d} \Omega\right)(q) S(q, \phi)$ scales with $\phi(1-\phi)$, whereas the scattering invariant of $n\left(\mathrm{~d} \sigma_{p} / \mathrm{d} \Omega\right)(q)$ just scales with $\phi$. This can be easily numerically verified for hard spheres using the analytical structure factor for a hard sphere potential in the Percus-Yevik approximation (Percus \& Yevick, 1958) and the form factor for a homogeneous sphere (Pedersen, 1997). The ratio between the scattering invariants is

$$
\frac{\int \frac{\mathrm{d} \sigma_{p}}{\mathrm{~d} \Omega}(q) S(q, \phi) q^{2} \mathrm{~d} q}{\int \frac{\mathrm{d} \sigma_{p}}{\mathrm{~d} \Omega}(q) q^{2} \mathrm{~d} q}=1-\phi .
$$

As the involved integrals cannot be solved analytically, we show in Fig. 1 that at least numerically equation (14) is valid for hard sphere colloids. This again shows that the scaling on the volume fraction, as done by Andersson et al. (2008), can be omitted when interparticle correlations are already included properly in the correlation function.

Aside from interparticle interactions, high volume fractions might lead to multiple scattering effects. Multiple scattering effects can be much easier to treat in spin-echo-based SANS or DF imaging than in classical SANS. Rekveldt (1996) has shown that instead of a linear dependency of the depolarization $P / P_{0}$ on the projected correlation function $\tilde{G}$ one has to use a simple exponential dependency as given in equations (1) and (2). This means that even in the presence of multiple 
scattering one can easily obtain from the experimental data the projected correlation function $\tilde{G}$, as already mentioned, only via material properties and it is independent of experimental parameters and effects like wavelength, sample thickness or multiple scattering effects.

In the literature often the normalized autocorrelation function $\gamma_{0}(r)=\tilde{\gamma}(r) / \tilde{\gamma}(0)$ is supplied, but only for a limited number of simple particle shapes. Before applying the transformation cycle we need to retrieve the unnormalized scattering length density contrast autocorrelation function $\tilde{\gamma}_{p}$ to obtain the correct absolute values for the differential scattering cross section $\left(\mathrm{d} \sigma_{p} / \mathrm{d} \Omega\right)(q)$. For a homogeneous single particle of volume $V_{p}$ and homogeneous scattering length density contrast $\Delta \eta$

$$
\tilde{\gamma}_{p}(0)=\int_{V_{p}}\left|\Delta \eta\left(\mathbf{r}^{\prime}\right)\right|^{2} \mathrm{~d} \mathbf{r}^{\prime}=\Delta \eta^{2} V_{p} .
$$

As for $N$ noninteracting identical homogeneous particles the differential scattering cross section in forward scattering is $I(0)=\left(N / V_{\mathrm{s}}\right) \Delta \eta^{2} V_{p}^{2}$ we get

$$
\int_{0}^{\infty} \gamma_{0}(r) 4 \pi r^{2} \mathrm{~d} r=V_{p}
$$

This helps to recover the unnormalized correlation function $\tilde{\gamma}(r)$ from the normalized correlation function $\gamma_{0}(r)$ as indicated in Fig. 2 at least for particles with a homogeneous scattering length density distribution. This first step is necessary to get the right interpretation and scaling of the isotropic three-dimensional Fourier transform as a differential scattering cross section per particle. This is important for the optional following averaging over a size distribution.

\section{Projected correlation function $\tilde{\mathbf{G}}(\boldsymbol{\delta})$}

Krouglov et al. (2003) and Andersson et al. (2008) showed that the projected correlation function $\tilde{G}(\delta)$ and the scattering

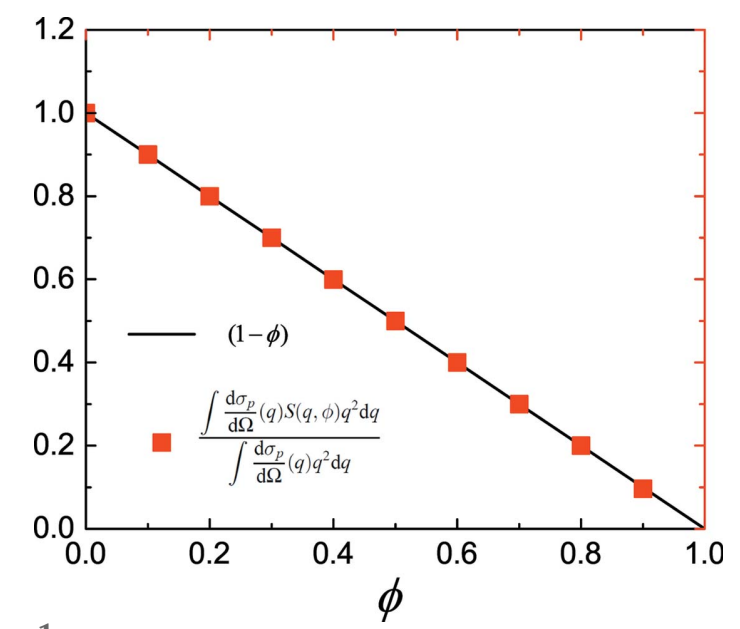

Figure 1

The ratio between the scattering invariants of a model of concentrated homogeneous spherical particles with a structure factor obtained for a hard sphere interaction potential in the Percus-Yevik approximation and the scattering invariant in the dilute limit. cross section $I(q)$ are for an isotropic scatterer related via a Hankel transform $\mathcal{H}$ :

$$
\tilde{G}(\delta)=\frac{1}{2 \pi} \int_{0}^{\infty} I(q) J_{0}(q \delta) q \mathrm{~d} q=\frac{1}{2 \pi} \mathcal{H}[I(q)](\delta) .
$$

The experimental measurable polarization $P_{\mathrm{s}}(\delta) / P_{0}(\delta)$ as a function of the spin-echo length $\delta$ and the visibility $V_{\mathrm{s}}\left(\xi_{\mathrm{GI}}\right) / V_{0}\left(\xi_{\mathrm{GI}}\right)$ as a function of the correlation length $\xi_{\mathrm{GI}}$ both depend in the same way on $\tilde{G}$ :

$$
\begin{aligned}
\frac{P_{\mathrm{s}}(\delta)}{P_{0}(\delta)} & =\exp \left\{t \lambda^{2}[\tilde{G}(\delta)-\tilde{G}(0)]\right\}, \\
\frac{V_{\mathrm{s}}\left(\xi_{\mathrm{GI}}\right)}{V_{0}\left(\xi_{\mathrm{GI}}\right)} & =\exp \left\{t \lambda^{2}\left[\tilde{G}\left(\xi_{\mathrm{GI}}\right)-\tilde{G}(0)\right]\right\},
\end{aligned}
$$

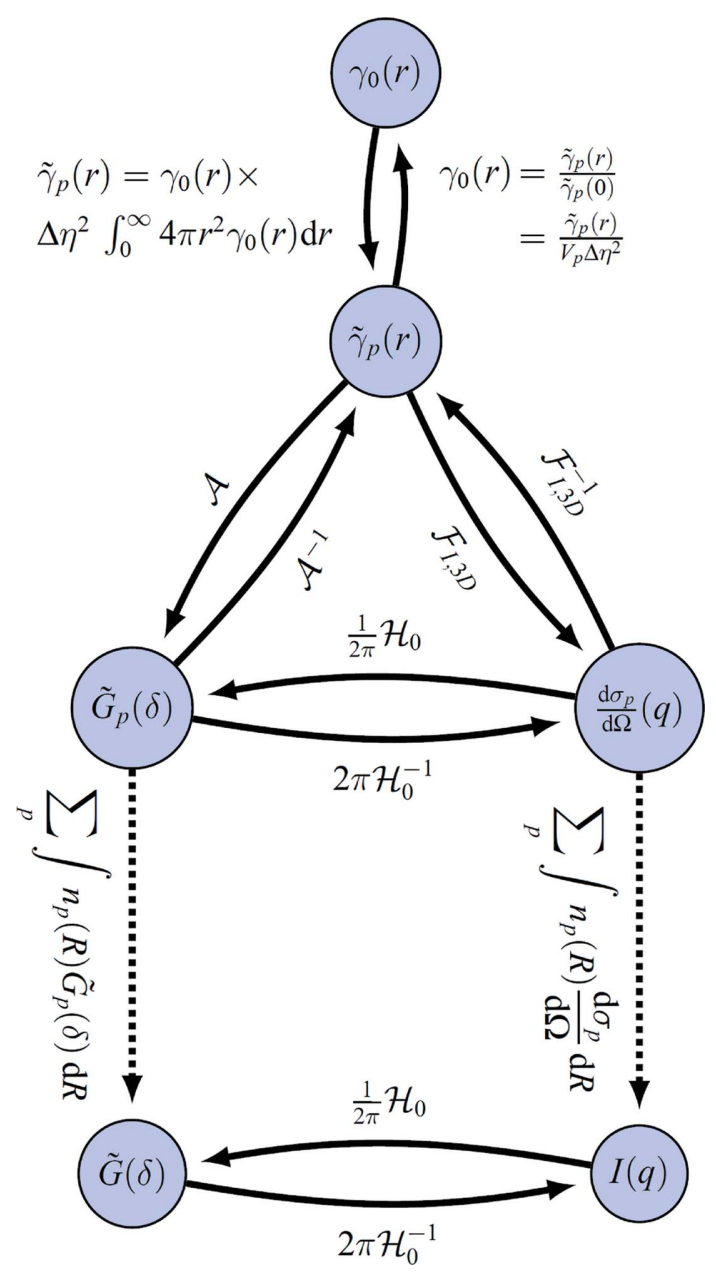

Figure 2

Transformation cycle between $\tilde{\gamma}(r), I(q)$ and $\tilde{G}(\delta)$ as implemented in the $S A S f i t$ software package. The averaging operator over the particle size is dotted, as this transformation is only allowed for non-interacting particles. In the case of monodisperse particles with interparticle interactions, equation (12) has to be applied first, which also requires solution of the Ornstein-Zernike equations. An additional size distribution would have to be included correctly in this step and unlike in the diagram shown here. Including a size distribution as shown in the diagram is a rough approximation and is known as the local monodisperse approximation'. 
where $\lambda$ is the wavelength of the incident beam and $t$ the sample thickness. Furthermore, the projected correlation function $\tilde{G}(\delta)$ is related to the density autocorrelation function via the Abel transform $\mathcal{A}$ by

$$
\tilde{G}(\delta)=\int_{\delta}^{\infty} \frac{2 r \tilde{\gamma}(r)}{\left(r^{2}-\delta^{2}\right)^{1 / 2}} \mathrm{~d} r=\mathcal{A}[\tilde{\gamma}(r)](\delta) .
$$

Andersson et al. (2008) applied a similar transformation cycle to that in Fig. 2. We first want to give for the transformation cycle as defined in Fig. 2 the missing mathematical proof on the basis of the $\mathcal{F H} \mathcal{A}$ cycle, which differs in the used Fourier transformation. In the $\mathcal{F H} \mathcal{A}$ cycle a one-dimensional Fourier transform is used, whereas in the Andersson cycle an isotropic three-dimensional Fourier transform is applied. The difference of the cycle introduced by Andersson from the one presented here is that no additional scaling like $\tilde{\gamma}(r) / \tilde{\gamma}(r=0)$ or $\tilde{G}(\delta) / \tilde{G}(\delta=0)$ or normalization via the correlation length $\xi=2 \int_{0}^{\infty} \gamma_{0}(r) \mathrm{d} r$ are required. This simplified transformation cycle makes it easier to implement a consistent analysis including a size distribution as done in the SASfit analysis software package.

\section{Relation to the Fourier-Hankel-Abel cycle}

As the Fourier transform used in the $\mathcal{F H} \mathcal{A}$ cycle is not the same as the one used in the transformation cycle of Andersson and Krouglov we first show where they differ, but we will also show that one cycle can be mathematically proven by the other, including the avoidance of any additional normalization.

The Fourier-Hankel-Abel $(\mathcal{F H} \mathcal{A})$ cycle as used in imaging is defined for circularly symmetric functions, i.e. functions $f: \mathbb{R}^{2} \rightarrow \mathbb{R}$ where $f(\mathbf{r})$ can be expressed as $f(r), r=|\mathbf{r}|$. The Fourier transform applied in the $\mathcal{F H} \mathcal{A}$ cycle is a one-dimensional transform $\int_{-\infty}^{\infty} f(r) \exp (i k r) \mathrm{d} r$, where $f(r)=f(-r)$ is assumed. In scattering we have spherically symmetric functions $f: \mathbb{R}^{3} \rightarrow \mathbb{R}$. Consequently, here we apply a threedimensional Fourier transform instead. That the transformation cycle is still valid will be shown first.

To start, the definitions of the Abel, Fourier and Hankel transforms are recapitulated. The Abel transform is a special case of the Radon transform in the sense that for the Abel transform the input function $f: \mathbb{R}^{2} \rightarrow \mathbb{R}$ is circularly symmetric, whereas the Radon transform takes arbitrary functions as input.

The Abel transform $\mathcal{A}: L^{2}\left(\mathbb{R}_{>0}, \mathbb{R}\right) \rightarrow L^{2}(\mathbb{R}, \mathbb{R})$ is defined as

$$
\mathcal{A}[f](y):=\int_{y}^{\infty} \frac{f(r) 2 r \mathrm{~d} r}{\left(r^{2}-y^{2}\right)^{1 / 2}} .
$$

The Abel transform can be interpreted as the (line integral) projection of $f$ onto the axis parallel to the $y$ axis located at $x=\infty$. For input functions $f$ decaying faster than $1 / r$ the Abel transform can be inverted. However, in the following proof the inversion of the Abel transform is deliberately avoided.
The Hankel transform $\mathcal{H}_{v}: L^{2}\left(\mathbb{R}_{>0}, \mathbb{R}\right) \rightarrow L^{2}\left(\mathbb{R}_{>0}, \mathbb{R}\right)$ of order $v$ is defined as

$$
\mathcal{H}_{\nu}[f](k):=\int_{0}^{\infty} f(r) J_{v}(k r) r \mathrm{~d} r
$$

where $J_{v}$ is the Bessel function of the first kind of order $v$. The Hankel transform is its own inverse for all $v, \mathcal{H}_{v}=\mathcal{H}_{v}^{-1}$.

The $n$-dimensional Fourier transform $\mathcal{F}_{n \mathrm{D}}: L^{2}\left(\mathbb{R}^{n}, \mathbb{C}\right) \rightarrow$ $L^{2}\left(\mathbb{R}^{n}, \mathbb{C}\right)$ is defined as

$$
\mathcal{F}_{n \mathrm{D}}[f](\mathbf{k}):=\int_{\mathbb{R}^{n}} f(\mathbf{r}) \exp (-i \mathbf{k} \cdot \mathbf{r}) \mathrm{d} \mathbf{r} .
$$

The inverse Fourier transform is similar to the Fourier transform: basically only the sign in the exponent changes and there is an additional constant factor of $(2 \pi)^{-n}$. In scattering science one often uses a different convention for defining the pairs of Fourier and Hankel transforms than in imaging. For example, Bracewell (1956) used the variable $\mathbf{s}=\mathbf{k} /(2 \pi)$, so that the transforms read as $\mathcal{F}_{n \mathrm{D}}^{(\mathrm{B})}[f](\mathbf{s})=\int_{\mathbb{R}^{n}} f(\mathbf{r}) \exp (-i 2 \pi \mathbf{s} \cdot \mathbf{r}) \mathrm{d} \mathbf{r}$ and $\mathcal{H}_{v}^{(\mathrm{B})}[f](s):=2 \pi \int_{0}^{\infty} f(r) J_{v}(2 \pi s r) r \mathrm{~d} r$. Choosing these definitions for the Hankel and Fourier transforms allows one to define the $\mathcal{F H} \mathcal{H}$ cycle without any additional factors of $2 \pi$ as in equation (3).

The Fourier transform of a radially symmetric function can be expressed as a Hankel transform (index I stands for isotropic and $n \mathrm{D}$ for the dimension):

$$
\mathcal{F}_{\mathrm{I}, n \mathrm{D}}[f](k)=\frac{(2 \pi)^{n / 2}}{k^{n / 2-1}} \int_{0}^{\infty} r^{n / 2-1} f(r) J_{n / 2-1}(k r) r \mathrm{~d} r,
$$

i.e.

$$
\mathcal{F}_{\mathrm{I}, n \mathrm{D}}[f](k)=\frac{(2 \pi)^{n / 2} \mathcal{H}_{v=n / 2-1}\left[f(r) r^{n / 2-1}\right](k)}{k^{n / 2-1}} .
$$

For the physically relevant cases $n=2$ and $n=3$, this yields

$$
\mathcal{F}_{\mathrm{I}, 2 \mathrm{D}}[f](k)=2 \pi \mathcal{H}_{v=0}[f](k)
$$

and

$$
\mathcal{F}_{\mathrm{I}, 3 \mathrm{D}}[f](k)=\frac{(2 \pi)^{3 / 2} \mathcal{H}_{v=1 / 2}\left[r^{1 / 2} f(r)\right](k)}{k^{1 / 2}} .
$$

Now that the three transforms are recalled, we can state the Fourier-Hankel-Abel cycle with the pairs of Hankel and Fourier conventions we have used:

$$
\mathcal{H}_{0} \circ 2 \pi \mathcal{F}_{1 \mathrm{D}} \circ \mathcal{A}=\mathcal{I} \Leftrightarrow \mathcal{A}=\mathcal{F}_{1 \mathrm{D}} \circ \mathcal{H}_{0} .
$$

In the second part of the above equation, we use the fact that the Fourier transform is its own inverse as the functions to transform are assumed to be symmetric. However, owing to the pair of Fourier transforms used here, we get a factor of $2 \pi$ as $\mathcal{F}_{1 \mathrm{D}} \circ \mathcal{F}_{1 \mathrm{D}}=(1 / 2 \pi) \mathcal{I}$ for symmetric functions.

The transformation cycle established first for SESANS uses an isotropic three-dimensional Fourier transform $\mathcal{F}_{\mathrm{I}, 3 \mathrm{D}}$ so that the cycle reads as

$$
\mathcal{A}=\mathcal{H}_{0} \circ \mathcal{F}_{\mathrm{I}, 3 \mathrm{D}} /(2 \pi)
$$


To prove the validity of this cycle, we refer to equation (28), which, combined with the above, yields

$$
\mathcal{A}=\mathcal{F}_{1 \mathrm{D}} \circ \mathcal{H}_{0}=\mathcal{H}_{0} \circ \mathcal{F}_{\mathrm{I}, 3 \mathrm{D}} /(2 \pi) .
$$

Left-multiplying ('composing') this equation with the (inverse) zero-degree Hankel transform gives

$$
\mathcal{F}_{\mathrm{I}, 3 \mathrm{D}}=2 \pi \mathcal{H}_{0} \circ \mathcal{F}_{1 \mathrm{D}} \circ \mathcal{H}_{0} .
$$

We prove this equation by mere calculation. To do so, we choose a suitable test function $\varphi$ (decaying faster than $1 / r$ ). For the left-hand side we write

$$
\mathcal{F}_{\mathrm{I}, 3 \mathrm{D}}[\varphi]\left(q^{\prime}\right)=4 \pi \int \frac{\sin \left(q^{\prime} r\right)}{q^{\prime}} \varphi(r) r \mathrm{~d} r .
$$

For the right-hand side we get

$$
\begin{aligned}
\mathcal{H}_{0} \circ 2 \pi \mathcal{F}_{1 \mathrm{D}} \circ \mathcal{H}_{0}[\varphi]\left(q^{\prime}\right)=\int_{0}^{\infty} J_{0}\left(q^{\prime} r^{\prime}\right) r^{\prime} \\
\quad \times\left\{2 \pi \int_{-\infty}^{\infty} \exp \left(-i q r^{\prime}\right)\left[\int_{0}^{\infty} J_{0}(q r) r \varphi(r) \mathrm{d} r\right] \mathrm{d} q\right\} \mathrm{d} r^{\prime} .
\end{aligned}
$$

Now we reorder the integrals:

$$
\begin{aligned}
& \mathcal{H}_{0} \circ 2 \pi \mathcal{F}_{1 \mathrm{D}} \circ \mathcal{H}_{0}[\varphi]\left(q^{\prime}\right) \\
& =2 \pi \int\left[\iint J_{0}\left(q^{\prime} r^{\prime}\right) r^{\prime} \exp \left(-i q r^{\prime}\right) J_{0}(q r) \mathrm{d} q \mathrm{~d} r^{\prime}\right] \varphi(r) r \mathrm{~d} r .
\end{aligned}
$$

If we compare this equation with equation (32), we see that it must hold that ( $\varphi$ is arbitrary)

$$
\int J_{0}\left(q^{\prime} r^{\prime}\right) r^{\prime} \int \exp \left(-i q r^{\prime}\right) J_{0}(q r) \mathrm{d} q \mathrm{~d} r^{\prime}=\frac{2 \sin \left(q^{\prime} r\right)}{q^{\prime}} .
$$

On the left side, we evaluate the first (inner) integral, which is the scaled one-dimensional Fourier transform of the zeroorder Bessel function:

$$
\mathcal{F}_{1 \mathrm{D}}\left[J_{0}(q r)\right]\left(r^{\prime}\right)=\frac{1}{|r|} \hat{J}_{0}\left(\frac{r^{\prime}}{r}\right)=\frac{2}{|r|\left[1-\left(r^{\prime} / r\right)^{2}\right]^{1 / 2}} \operatorname{rect}_{[-1,1]}\left(\frac{r^{\prime}}{r}\right),
$$

where we use the general scaling property of $\mathcal{F}_{1 \mathrm{D}}$ : $\mathcal{F}_{1 \mathrm{D}}[f(\tau t)](\omega)=(1 /|\tau|) \hat{f}(\omega / \tau)$. $\operatorname{rect}_{[-1,1]}$ is the rectangular function, which is 1 for arguments between -1 and 1 and zero otherwise. Having calculated the inner integral, the outer integral of equation (35) is hence the zero-degree Hankel transform of equation (36), right side. The Hankel transform of $\hat{J}_{0}$ is a sinc function and, under scaling, the Hankel transform changes as $\mathcal{H}_{0}[f(\lambda r)](q)=\lambda^{-2}\left[\mathcal{H}_{0} f\right](q / \lambda)$. Hence (the scaling now is $1 / r$ )

$$
\frac{1}{r} \mathcal{H}_{0}\left[\hat{J}_{0}\left(\frac{r^{\prime}}{r}\right)\right]\left(q^{\prime}\right)=\frac{2 \sin \left(q^{\prime} r\right)}{q^{\prime}} .
$$

This proves the validity of the $\mathcal{A}=\mathcal{H}_{0} \circ \mathcal{F}_{\mathrm{I}, 3 \mathrm{D}} /(2 \pi)$ cycle and can be cross-checked easily with any computer algebra software package on, for example, the function $\tilde{\gamma}_{\mathrm{DAB}}(r)=$ $8 \pi \Delta \eta^{2} \xi^{3} \exp (-r / \xi)$, which is the unnormalized correlation function for a random phase of an inhomogeneous solid as introduced by Debye \& Bueche (1949). Using the definition of the projected correlation function from equation (20), which reads $\tilde{G}(\delta)=\mathcal{A}[\tilde{\gamma}(r)](\delta)$, allows one to perform both routes as given by equation (30) analytically as well as the Abel transform itself:

$$
\mathcal{A}\left[\tilde{\gamma}_{\mathrm{DAB}}(r)\right](\delta)=16 \pi \Delta \eta^{2} \xi^{3} \delta \mathrm{K}_{1}(\delta / \xi),
$$

$$
\begin{aligned}
\mathcal{F}_{1 \mathrm{D}} \circ \mathcal{H}_{0}\left[\tilde{\gamma}_{\mathrm{DAB}}(r)\right](\delta) & =\int_{-\infty}^{\infty} \exp (-i q \delta) \int_{0}^{\infty} \tilde{\gamma}_{\mathrm{DAB}}(r) J_{0}(q r) r \mathrm{~d} r \mathrm{~d} q \\
& =\int_{-\infty}^{\infty} \exp (-i q \delta) \frac{8 \pi \Delta \eta^{2} \xi^{5}}{\left(1+q^{2} \xi^{2}\right)^{3 / 2}} \mathrm{~d} q \\
& =16 \pi \Delta \eta^{2} \xi^{3} \delta K_{1}(\delta / \xi)
\end{aligned}
$$

$$
\begin{aligned}
\mathcal{H}_{0} \circ \frac{1}{2 \pi} \mathcal{F}_{\mathrm{I}, 3 \mathrm{D}}\left[\tilde{\gamma}_{\mathrm{DAB}}(r)\right] & (\delta) \\
& =\int_{0}^{\infty} J_{0}(q \delta) q \int_{0}^{\infty} \tilde{\gamma}_{\mathrm{DAB}}(r) \frac{\sin q r}{q r} 2 r^{2} \mathrm{~d} r \mathrm{~d} q \\
& =\int_{0}^{\infty} J_{0}(q \delta) q \frac{32 \pi \xi^{6}}{\left(1+q^{2} \xi^{2}\right)^{2}} \mathrm{~d} q \\
& =16 \pi \Delta \eta^{2} \xi^{3} \delta \mathrm{K}_{1}(\delta / \xi) .
\end{aligned}
$$

$K_{1}$ is the modified Bessel function of second kind and first order.

\section{5. $\tilde{\mathbf{G}}(\delta)$ and absolute scale in SASfit}

The function $\tilde{G}(\delta)$ has been implemented in the open-source software package SASfit (Breßler et al., 2015). SASfit allows users to fit experimental small-angle scattering data which can be described in the dilute case as a linear combination of the scattering contribution of particles of different shape including a size distribution. Up to now in the package only form factors have been supplied. The function for the form factor in most cases describes the scattering cross section of a single particle. The scattering cross section of a single object needs to be scaled with the number density of particles in the beam to get the absolute units of the differential scattering cross section. This has been realized by including a size distribution to describe a polydisperse system, i.e.

$$
I(q)=\sum_{p} \int_{0}^{\infty} n_{p}(R) \frac{\mathrm{d} \sigma_{p}}{\mathrm{~d} \Omega}(q, R) \mathrm{d} R .
$$

The scattering can be described by a sum of different types of particles labelled with $p$, described by their differential scattering cross section $\mathrm{d} \sigma_{p} / \mathrm{d} \Omega$ and their size distribution $n_{p}(R)$. The size distribution is normalized to the number density $N_{p}$ so that

$$
N_{p}=\int_{0}^{\infty} n_{p}(R) \mathrm{d} R
$$

$R$ does not need to be a radius. It can be any characteristic size parameter of the particle. 
Also for the projected correlation function we can describe the total correlation function of a system of noninteracting particle species by

$$
\tilde{G}(\delta)=\sum_{p} \int_{0}^{\infty} n_{p}(R) \tilde{G}_{p}(\delta, R) \mathrm{d} R,
$$

where $\tilde{G}_{p}(\delta, R)$ is the projected correlation function of a single particle of type $p$ and size $R$. The averaging over the size distribution cannot be done directly on a normalized projected correlation function as the normalized function would not scale with the particle volume $V_{p}$ at $\delta=0$. Therefore we profit here from the avoidance of any normalization to 1 .

A simple and numerically efficient way to supply the projected correlation function in $S A S f i$ is to implement known analytical expressions for $\tilde{G}_{p}(\delta, R)$ of single objects. However, $\tilde{G}_{p}(\delta, R)$ has been calculated up to now only for a few particle shapes. To extend the number of available shapes an operator which can be either the identity operator $\mathcal{I}$ or $(2 \pi)^{-1}$ times the Hankel transform, or more precisely $(1 / 2 \pi)\{\mathcal{H}[I(q)](\delta)-\mathcal{H}[I(q)](0)\}$, has been introduced in the software package. By activating one or the other operator one can quickly convert any known scattering function into a projected correlation function, i.e. into $\tilde{G}(\delta)-\tilde{G}(0)$, as this difference is actually the quantity to which SESANS, SEMSANS and DF imaging are sensitive [equations (18) and (19)]. For the efficient numerical calculation of the Hankel transform the algorithm of Ooura \& Mori (1991) has been used.

The two equations (18) and (19) also show that the experimental quantities like polarization or visibility have to be transformed to get

$$
\tilde{G}(\delta)-\tilde{G}(0)=\frac{1}{t \lambda^{2}} \ln \left[P_{\mathrm{s}}(\delta) / P_{0}(\delta)\right],
$$

which is independent of instrument parameters and has a unit of $1 /$ volume. To be consistent in units in the transformation cycle shown in Fig. 2 one should keep the same units for

$$
[\lambda]=[\delta]=\left[\xi_{\mathrm{GI}}\right]=[R]=[1 / q],
$$

i.e. $\mathrm{nm}$ or $\AA$, and

$$
[t]=[1 / I(q)],
$$

i.e. $\mathrm{cm}$. By this the projected correlation function will have the unit $[\tilde{G}(\delta)]=[q]^{2}[I(q)]$, i.e. $[\tilde{G}(\delta)]=\mathrm{nm}^{-2} \mathrm{~cm}^{-1}$ or $[\tilde{G}(\delta)]=$ $\AA^{-2} \mathrm{~cm}^{-1}$.

Similarly the projected correlation function $\tilde{G}_{p}(\delta)$ of a single particle is dimensionless and the differential scattering cross section $\left(\mathrm{d} \sigma_{p} / \mathrm{d} \Omega\right)(q)$ of a single particle has units of area. However, in both functions the scattering length density contrast $\Delta \eta$ in units of $1 /$ area and the parameter describing the size of the particle, which has a length unit, are used. Here too the transformation cycle is consistent in units if they are chosen as in equation (45). The units of the scattering length density $\Delta \eta$ determine just the scaling factor of the unit for the normalization factor $N_{p}$ in the distribution function [equation (42)]. More details can be found in the SASfit manual.

\section{6. $\tilde{\mathbf{G}}(\delta)$ for the generalized Gaussian coil model}

The Hankel transform of the scattering intensity $I(q)$ for $\delta=0$ corresponds to the integrated intensity $\mathcal{H}_{0}[I(q)](0)=$ $\int_{0}^{\infty} q I(q) \mathrm{d} q$, which is only finite for scattering curves decaying faster than $q^{-2}$ for large $q$ values. For polymers at the $\Theta$ temperature ${ }^{1}$ (Flory, 1974) the scattering intensity decays with $q^{-2}$ and the Hankel transform $\mathcal{H}_{0}\left[I_{\text {Gauss }}(q)\right](0)=\infty$ therefore diverges at $\delta=0$. In practice this does not happen, firstly because of the limited detector size and therefore a truncation of the Hankel transform and secondly because in the case of purely elastic scattering the maximum $q$ value cannot exceed $2 k_{0}>q$, which corresponds to backscattering. The limited acceptance angle is discussed in more detail by Kusmin et al. (2017), along with several other effects like wavelength resolution, detector resolution etc.

$S A S f i t$ can read SESANS data files from TU Delft. These data also contain the maximum measured $q_{\max }$ values. This $q_{\max }$ limit can be used in SASfit as a truncation of the Hankel transform for the analysis of SESANS data, which allows us also to use form factors decaying slower than $q^{-2}$. The effect of a limited detector size and therefore a maximum detected $q$ value might become significant in the case of a very slowly decaying scattering function, even though the corresponding structure itself is large compared to the $q_{\max }$ limit. We want to show this effect on a generalization (gGc) of the classical Gaussian coil model for polymers, which decays at large $q$ values with varying power law, depending on the solvent quality. The analytical solution for the scattering intensity is given according to Hammouda $(1993,2012,2016)$ in the limit of long polymer chains by

$$
\begin{aligned}
I(q) & =I_{0} 2 \int_{0}^{1} \mathrm{~d} x(1-x) \exp \left[-\frac{q^{2} R_{\mathrm{g}}^{2}}{6}(2 v+1)(2 v+2) x^{2 v}\right] \\
& =I_{0}\left[\frac{1}{v U^{1 / 2 v}} \gamma\left(\frac{1}{2 v}, U\right)-\frac{1}{v U^{1 / v}} \gamma\left(\frac{1}{v}, U\right)\right]
\end{aligned}
$$

with the modified variable

$$
U=(2 v+1)(2 v+2) q^{2} R_{\mathrm{g}}^{2} / 6
$$

and the lower incomplete Gamma function $\gamma(a, x)=$ $\int_{0}^{x} \mathrm{~d} t t^{a-1} \exp (-t) . R_{\mathrm{g}}$ denotes the radius of gyration. $v$ is the excluded volume parameter from the Flory mean field theory and typical values are $v=1 / 3$ for partially precipitated polymers in poor solvents, $v=1 / 2$ for thermally relaxed polymers in $\Theta$ solvents and $v=3 / 5$ for swollen polymers in good solvents. We start from equation (47) on which we apply the Hankel transform. After changing the order of integration over $\mathrm{d} x$ and $\mathrm{d} q$, we can solve both integrals analytically with the result

\footnotetext{
$\mathbf{1}$ Thermodynamically, the $\Theta$ temperature of a solvent is defined by a vanishing excess chemical potential between polymer and solvent. In practice, at this point the polymer shows a conformation which can be described by a random walk behaviour. At this temperature the second osmotic virial coefficient is zero and marks the temperature transition between a good and poor solvent.
} 


$$
\begin{gathered}
\tilde{G}_{\mathrm{gGc}}(\delta)=\frac{I_{0}}{v \delta^{2} \pi}\left[w^{1 / 2 v} \Gamma\left(1-\frac{1}{2 v}, w\right)-w^{1 / v} \Gamma\left(1-\frac{1}{v}, w\right)\right] \\
w=\frac{3}{4} \frac{\delta^{2}}{\left(2 v^{2}+3 v+1\right) R_{\mathrm{g}}^{2}}
\end{gathered}
$$

where $\Gamma(a, x)=\int_{x}^{\infty} \mathrm{d} t t^{a-1} \underset{\tilde{G}}{\exp }(-t)$ is the upper incomplete Gamma function. The limit $\tilde{G}_{\mathrm{gGc}}(0)$ is only finite for $v \in\left(0, \frac{1}{2}\right)$ :

$$
\tilde{G}_{\mathrm{gGc}}(0)=I_{0} \frac{3}{4 \pi} \frac{1}{\left(4 \nu^{4}-5 v^{2}+1\right) R_{\mathrm{g}}^{2}} \quad \text { for } \quad v \in\left(0, \frac{1}{2}\right),
$$

which is consistent with the above statement that a classical Gaussian polymer with $v=\frac{1}{2}$ does not have a finite Hankel transform for $\delta=0$.

Fig. 3 shows the calculation of $\tilde{G}(\delta)-\tilde{G}(0)$ for polydisperse spheres with a lognormal distribution calculated via Hankel transformation of the scattering intensity and by integrating the projected correlation function of spheres with a size distribution. Also for the generalized Gaussian coil model the projected correlation function has been calculated via equation (50) as well as by $(2 \pi)^{-1}$ times the Hankel transform of



(a)

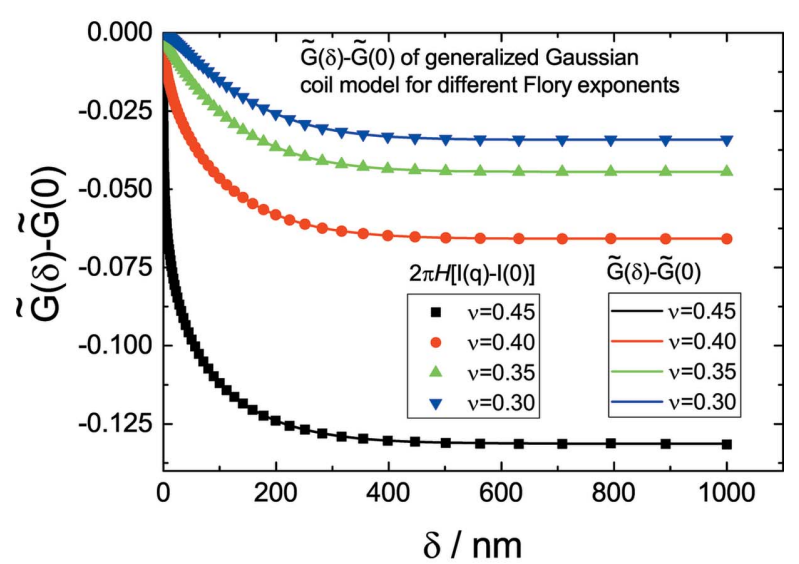

(b)

Figure 3

(a) Test that the plugin functions in SASfit for the projected correlation function $\tilde{G}(\delta)-\tilde{G}(0)$ for spheres with a size distribution [equation (43)] and the Hankel transform of the scattering intensity according to equation (41) with the same size distribution parameters are identical. (b) Calculation of $\tilde{G}_{\mathrm{gGc}}(\delta)$ by equation (50) and by the numerical Hankel transform of the intensity given in equation (48). equation (48). In both cases the two calculation routes lead to the same results, as shown in Fig. 3.

In Fig. 4 we show the influence of a limited detector size, which has been included in the Hankel transform by performing the integration only up to $q_{\max }$. At the SESANS setup in Delft this corresponds to a typical value of $q_{\max }=0.5 \mathrm{~nm}^{-1}$. In Fig. 4(a) the scattering curves of a polymer described by the generalized Gaussian coil model for different excluded volume parameters ranging from $v=0.3$ to $v=0.45$ are shown. The corresponding projected correlation functions are calculated firstly by the untruncated Hankel transform (solid line) and secondly by a truncated one at $q_{\max }=0.5 \mathrm{~nm}^{-1}$ (symbols). The truncation is marked in the plot of the scattering intensity by a dashed line. The models are scaled so that they all have the same $\tilde{G}(0)$ for the untruncated Hankel transform. The effect of a slowly decaying scattering function, i.e. larger excluded volume parameter $v$, mainly affects the absolute value of $\tilde{G}(0)$ and the shape of $\tilde{G}(\delta)$

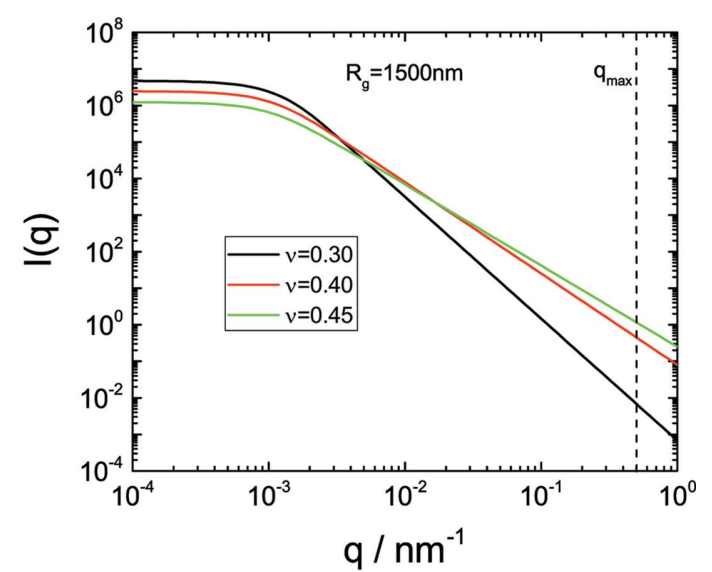

(a)



(b)

Figure 4

Effect of finite detector size and therefore a maximum detected $q$ value for the case of a generalized Gaussian coil model with varying decaying potential laws at large $q$ values. (a) The scattering curves of a polymer in different solvent qualities with an excluded volume parameter in the range $v=0.3-0.45$ but the same radius of gyration of $1.5 \mu \mathrm{m}$. The scattering curves are scaled so that they have the same value for $\tilde{G}(0)$. (b) The projected correlation functions $\tilde{G}(\delta)-\tilde{G}(0)$ calculated via a Hankel transform of the scattering curve over all $q$ values (line) and secondly only up to the maximum detectable $q_{\max }=0.5 \mathrm{~nm}^{-1}$ (symbols). 
at very small spin-echo lengths $\delta$ as there the truncation in $q$ is most significant.

\section{Summary and conclusion}

An extended version of SASfit (version 0.94.9), available at http://sasfit.org, has been published. The new version is now able to analyse and fit projected correlation functions obtained from spin-echo-based SANS or DF imaging. For this purpose the ability to read data files with a SESANS format has been introduced. The reading routine automatically converts the polarization into $\tilde{G}(\delta)-\tilde{G}(0)=\left(1 / t \lambda^{2}\right) \times$ $\ln \left[P_{\mathrm{s}}(\delta) / P_{0}(\delta)\right]$ [equation (44)]. To fit the projected correlation function one can either choose directly an analytical model for it or use an optional Hankel transform of the small-angle scattering intensity. Up to now only very few projected correlation functions have been supplied in the SASfit package, but the list can be easily extended by the provided plugin mechanism. Also these models can be combined with a size distribution in the same way as form factors used for standard small-angle scattering data analysis. A simplified transformation cycle has been established, which relates the spherically symmetric correlation function $\tilde{\gamma}(r)$, the projected correlation function $\tilde{G}(\delta)$ and the differential small-angle scattering cross section $\mathrm{d} \sigma / \mathrm{d} \Omega$ without any additional scaling or normalization. This is an advantage especially in those cases where the correlation length cannot be calculated, because the Hankel transform for $\delta=0$ of the scattering model is divergent, for example for scattering models of polymers with a scattering behaviour decaying slower than $q^{-2}$. In such cases the measurable part of the projected correlation function still can be calculated by truncating the Hankel transform at a maximum scattering vector $q_{\text {max }}$ determined by the solid angle covered by the detector and the used wavelength. The avoidance of the normalization also simplifies the averaging over a size distribution which can be performed without additional weighting factors. An anisotropic scatterer can be handled for analysing small-angle scattering data, but the Hankel transforms of such scattering models are not treated properly in the package yet. They should not be used in the present version for calculating the projected correlation functions via small-angle scattering intensity. To be able to compare small-angle scattering data and projected correlation function model parameters on an absolute scale including number densities of particles one just needs to keep the same units for some model and experimental parameters [equations (45) and (46)].

\section{Acknowledgements}

Many thanks are given to Wim Bouwman from the Delft University of Technology for very fruitful discussions, many useful hints and testing the numerical algorithm.

\section{References}

Andersson, R., van Heijkamp, L. F., de Schepper, I. M. \& Bouwman, W. G. (2008). J. Appl. Cryst. 41, 868-885.
Bomont, J.-M. (2008). Recent Advances in the Field of Integral Equation Theories: Bridge Functions and Applications to Classical Fluids, ch. 1, pp. 1-84. Hoboken: John Wiley and Sons.

Bracewell, R. (1956). Aust. J. Phys. 9, 198-217.

Bracewell, R. N. (1990). Science, 248, 697-704.

Bracewell, R. N. (2000). The Fourier Transform and its Applications. New York: McGraw-Hill.

Breßler, I., Kohlbrecher, J. \& Thünemann, A. F. (2015). J. Appl. Cryst. 48, 1587-1598.

Caccamo, C. (1996). Phys. Rep. 274, 1-105.

Debye, P. \& Bueche, A. M. (1949). J. Appl. Phys. 20, 518-525.

Flory, P. (1974). Nobel Lecture, 11 December 1974, http:// nobelprize.org/nobel_prizes/chemistry/laureates/1974/flory-lecture.pdf.

Grünzweig, C., David, C., Bunk, O., Dierolf, M., Frei, G., Kühne, G., Kohlbrecher, J., Schäfer, R., Lejcek, P., Rønnow, H. M. R. \& Pfeiffer, F. (2008). Phys. Rev. Lett. 101, 025504.

Hammouda, B. (1993). SANS from Homogeneous Polymer Mixtures: A Unified Overview, pp. 87-133. Berlin, Heidelberg: Springer.

Hammouda, B. (2012). Macromol. Theory Simul. 21, 372-381.

Hammouda, B. (2016). J. Res. NIST, 121, 139-164.

Krouglov, T., de Schepper, I. M., Bouwman, W. G. \& Rekveldt, M. T. (2003). J. Appl. Cryst. 36, 117-124.

Kruglov, T. (2005). J. Appl. Cryst. 38, 716-720.

Kusmin, A., Bouwman, W., van Well, A. \& Pappas, C. (2017). Nucl. Instrum. Methods Phys. Res. Sect. A, 856, 119-132.

Li, F., Parnell, S. R., Bai, H., Yang, W., Hamilton, W. A., Maranville, B. B., Ashkar, R., Baxter, D. V., Cremer, J. T. \& Pynn, R. (2016). J. Appl. Cryst. 49, 55-63.

Li, X., Shew, C., Liu, Y., Pynn, R., Liu, E., Herwig, K. W., Smith, G. S., Robertson, J. L. \& Chen, W. (2010). J. Chem. Phys. 132, 174509.

Li, X., Shew, C., Liu, Y., Pynn, R., Liu, E., Herwig, K. W., Smith, G. S., Robertson, J. L. \& Chen, W. (2011). J. Chem. Phys. 134, 094504.

Ooura, T. \& Mori, M. (1991). J. Comput. Appl. Math. 38, 353-360.

Pedersen, J. S. (1994). J. Appl. Cryst. 27, 595-608.

Pedersen, J. S. (1997). Adv. Colloid Interface Sci. 70, 171-210.

Percus, J. K. \& Yevick, G. J. (1958). Phys. Rev. 110, 1-13.

Pfeiffer, F., Bech, M., Bunk, O., Kraft, P., Eikenberry, E. F., Brönnimann, C., Grünzweig, C. \& David, C. (2008). Nat. Mater. 7, 134-137.

Pfeiffer, F., Grünzweig, C., Bunk, O., Frei, G., Lehmann, E. \& David, C. (2006). Phys. Rev. Lett. 96, 215505.

Pfeiffer, F., Weitkamp, T., Bunk, O. \& David, C. (2006). Nat. Phys. 2, 258-261.

Price, D. L. \& Fernandez-Alonso, F. (2013). Neutron Scattering Fundamentals, Experimental Methods in the Physical Sciences, Vol. 44, edited by F. Fernandez-Alonso \& D. L. Price, pp. 1-136. Amsterdam: Elsevier/Academic Press.

Rekveldt, M. (1996). Nucl. Instrum. Methods Phys. Res. Sect. B, 114, 366-370.

Rekveldt, M. T., Bouwman, W. G., Kraan, W. H., Uca, O., Grigoriev, S. V., Habicht, K. \& Keller, T. (2003). Elastic Neutron Scattering Measurements Using Larmor Precession of Polarized Neutrons, ch. 2, pp. 87-99. Berlin, Heidelberg: Springer.

Sales, M., Plomp, J., Habicht, K. \& Strobl, M. (2015). J. Appl. Cryst. 48, 92-96.

Strobl, M. (2014). Sci. Rep. 4, 7243.

Strobl, M., Betz, B., Harti, R. P., Hilger, A., Kardjilov, N., Manke, I. \& Gruenzweig, C. (2016). J. Appl. Cryst. 49, 569-573.

Strobl, M., Grünzweig, C., Hilger, A., Manke, I., Kardjilov, N., David, C. \& Pfeiffer, F. (2008). Phys. Rev. Lett. 101, 123902.

Strobl, M., Sales, M., Plomp, J., Bouwman, W. G., Tremsin, A. S., Kaestner, A., Pappas, C. \& Habicht, K. (2015). Sci. Rep. 5, 16576.

Strobl, M., Tremsin, A. S., Hilger, A., Wieder, F., Kardjilov, N., Manke, I., Bouwman, W. G. \& Plomp, J. (2012). J. Appl. Phys. 112, 014503.

Strobl, M., Wieder, F., Duif, C., Hilger, A., Kardjilov, N., Manke, I. \& Bouwman, W. (2012). Phys. B Condens. Matter, 407, 4132-4135.

Vrij, A. (1979). J. Chem. Phys. 71, 3267-3270. 